\title{
On the Brauer-Manin obstruction for zero-cycles on curves
}

by

\author{
Dennis Eriksson (Tokyo) and Victor Scharaschkin (Brisbane)
}

1. Brauer-Manin obstruction. For the purposes of this paper, a $v a-$ riety over a field $k$ is a separated scheme of finite type over $k$. We first recall the definition of the Brauer-Manin obstruction. Henceforth the symbols $X$ and $U$ will be used to denote varieties over a field $k$, and in case they are mentioned together, $U$ is a dense open set of $X$.

Suppose $k$ is perfect. Set $\operatorname{Br} X:=\mathrm{H}_{\mathrm{et}}^{2}\left(X, \mathbb{G}_{m}\right)$. By functoriality, an $L$ point (for $L / k$ a field extension) Spec $L \rightarrow X$ defines a homomorphism $\operatorname{Br} X \rightarrow \operatorname{Br} L$. Furthermore, if $L / k$ is finite, we can take corestriction $\operatorname{Br} L \stackrel{\text { Cores }}{\longrightarrow}$ Br $k$. Hence, by extending by linearity, we obtain a pairing

$$
Z_{0}(X) \times \operatorname{Br} X \rightarrow \operatorname{Br} k .
$$

Here $Z_{0}(X)$ denotes the group of zero-cycles on $X$. Now, let $k$ be a number field, and set $k_{v}$ to be the completion of $k$ at a place $v$. For a $k$-variety $X$, we denote $X \times_{k} k_{v}$ by $X_{v}$, and $X \times_{k} \bar{k}$ by $\bar{X}$ for a separable closure $\bar{k}$ of $k$. Denote by $Z_{0}^{\mathrm{a}}(X)$ the group $\prod_{v} Z_{0}\left(X_{v}\right)$. Suppose for the purposes of the introduction that $X$ is a connected smooth, geometrically integral variety. In the case $B$ is a subgroup of the unramified Brauer group $\mathrm{Br}_{\mathrm{nr}} X$ (the Brauer group of any smooth compactification of $X$; it is a birational invariant, cf. [5, Théorème 7.4]), it is possible to show that we can define a pairing

$$
Z_{0}^{\mathrm{a}}(X) \times B \rightarrow \mathbb{Q} / \mathbb{Z}
$$

as follows: Given a zero-cycle $\left(z_{v}\right)_{v}$ and an element $\alpha \in B$, one obtains elements $\left(\alpha\left(z_{v}\right)_{v}\right) \in \prod_{v} \mathrm{Br} k_{v}$ by evaluating as in (1). By local class field theory we have injections $i_{v}: \operatorname{Br} k_{v} \hookrightarrow \mathbb{Q} / \mathbb{Z}$ and one shows that since $\alpha \in$ $\operatorname{Br}_{\mathrm{nr}} X$, the element $\left\langle\left(z_{v}\right), \alpha\right\rangle=\sum_{v} i_{v}\left(\alpha\left(z_{v}\right)\right)$ is a finite sum and thus well defined.

2000 Mathematics Subject Classification: Primary 14H25; Secondary 14C15, 11G35.

Key words and phrases: Albanese, Brauer-Manin obstruction, curves, Suslin homology, zero-cycles. 
Now, one puts, for $B \subseteq \operatorname{Br}_{\mathrm{nr}} X$,

$$
Z_{0}^{\mathrm{a}}(X)^{B}=\left\{\left(z_{v}\right) \in Z_{0}^{\mathrm{a}}(X) \mid \forall v, \operatorname{deg} z_{v}=1,\left\langle\left(z_{v}\right), \alpha\right\rangle=0, \forall \alpha \in B\right\} .
$$

The corestriction map is the identity on the level of $\mathbb{Q} / \mathbb{Z}$ for non-archimedean places (see [15, XI, Prop. 2,ii) and XIII, Théorème 1]). Using this together with the fundamental short exact sequence of class field theory,

$$
0 \rightarrow \mathrm{Br} k \rightarrow \bigoplus_{v} \operatorname{Br} k_{v} \rightarrow \mathbb{Q} / \mathbb{Z} \rightarrow 0,
$$

one shows that $Z_{0}(X)^{1}$, the zero-cycles of degree one, are indeed included in the above defined set $Z_{0}^{\mathrm{a}}(X)^{B}$. This follows in a way completely analogous to the case of points (see Manin [8]). For the same reasons, the above pairing with $\mathrm{Br}_{0} k$, i.e. the image of $\operatorname{Br} k$ in $\operatorname{Br} X$, is seen to be zero. If the set $Z_{0}^{\text {a }}(X)^{B}$ is non-empty, we say there is no obstruction associated to $B$ for existence of zero-cycles of degree one. Now let $\mathcal{V}$ be a class of smooth, geometrically integral varieties with an assignment $B=B(X) \subseteq \mathrm{Br}_{\mathrm{nr}} X$ for any $X \in \mathcal{V}$. If for all $X$ in $\mathcal{V}$ we have

$$
Z_{0}^{\mathrm{a}}(X)^{B} \neq \emptyset \Rightarrow Z_{0}(X)^{1} \neq 0
$$

then we say the Brauer-Manin obstruction is the only one to the existence of zero-cycles of degree one associated to $B$. Let $\mathrm{Br}_{1} X=\operatorname{ker}[\operatorname{Br} X \rightarrow \operatorname{Br} \bar{X}]$. The group

$$
\mathrm{Б}(X):=\operatorname{ker}\left[\operatorname{Br}_{1} X \rightarrow \prod_{v} \operatorname{Br}_{1} X_{v} / \mathrm{Br} k_{v}\right]
$$

is the group of (algebraic) locally constant elements of the Brauer group. Whenever $X$ is a proper geometrically integral variety, the quotient of $\mathrm{L}(X)$ by the image $\mathrm{Br}_{0} k$ of $\mathrm{Br} k$ comes with a canonical isomorphism

$$
\mathrm{Б}(X) / \operatorname{Br}_{0}(k) \simeq \amalg^{1}(\operatorname{Pic}(\bar{X}))=\operatorname{ker}\left(\mathrm{H}^{1}(k, \operatorname{Pic}(\bar{X})) \rightarrow \prod_{v} \mathrm{H}^{1}\left(k_{v}, \operatorname{Pic}\left(\bar{X}_{v}\right)\right)\right)
$$

via the Hochschild-Serre spectral sequence (see [18, Corollary 2.3.9, the case $M=\mathbb{Z}]$; this is an isomorphism essentially because $\mathrm{H}^{3}\left(k, \mathbb{G}_{m}\right)=0$ for local and global fields). Here and henceforth $\mathrm{H}^{i}(k, M)$ denotes étale cohomology, which reduces to Galois cohomology of $\Gamma_{k}=\mathrm{Gal}(\bar{k} / k)$ with values in $M$. By Lemma 3.5 below, $\mathrm{\textrm {C }}(X) \subseteq \mathrm{Br}_{1}(X)$ is unramified in the sense that it does not depend on the choice of smooth compactification.

Let $X$ be a smooth quasi-projective variety defined over a perfect field $k$. Denote the semi-Albanese variety of $X$ by $\mathrm{SAlb}_{X}^{0}$. There is a certain torsor $\mathrm{SAlb}_{X}^{1}$ under $\mathrm{SAlb}_{X}^{0}$, universal with respect to morphisms from $X$ to torsors under semiabelian varieties (see Definition 3.1). The period, $P=P_{X}$, of $X$ is defined as the order of $\left[\mathrm{SAlb}_{X}^{1}\right]$ in $\mathrm{H}^{1}\left(k, \mathrm{SAlb}_{X}^{0}\right)$. In Theorem 4.2 of Section 4 we give another characterization of the period in terms of Suslin homology (see Appendix). The index $I=I_{X}$ of a variety $X$ over a field $k$ is defined to 
be the least positive degree of a zero-cycle on $X$ with respect to $k$. Define the generic period $\widetilde{P}$ as the supremum of $P_{U}$ over all open non-empty subsets $U$ of $X$. Note that $P_{U} \mid I_{U}$ and it is well known that the index of an open subset of $X$ is the same as that of $X$ (see [3, "Complément"]). Thus we see that all $P_{U}$ are bounded by $I$, so the supremum exists. Moreover, $\widetilde{P} \mid I$.

Our first result is a stronger version of a theorem originally due to S. Saito [13] (stronger, because here we only need the conjecturally finite group $\mathrm{B}(C) / \mathrm{Br}_{0} k$ as opposed to the whole Brauer group). S. Saito's theorem has also been reproved by Colliot-Thélène in [2].

THEOREM 1.1. Let $C$ be a smooth connected projective curve over a number field $k$, let $A$ be its Jacobian, and assume that $\amalg^{1}(A)$ is finite. Then the obstruction associated to $\mathrm{Б}(C)$ for zero-cycles of degree one is the only one:

$$
\text { if } Z_{0}^{\mathrm{a}}(C)^{\mathrm{B}(C)} \neq \emptyset \text { then } I=1 .
$$

We have an obvious corollary which does not involve the group $Z_{0}^{\text {a }}$ (see introduction):

COROllary 1.2. Let $C$ be as above and keep the same assumptions. Then if $C$ has no Brauer-Manin obstruction associated to $\mathrm{Б}(C)$ for rational points, then $C$ has a zero-cycle of degree one, i.e.

$$
\text { if } C\left(\mathbb{A}_{k}\right)^{\mathrm{E}(C)} \neq \emptyset \text { then } I=1 \text {. }
$$

For general projective, smooth, geometrically integral $k$-varieties ColliotThélène (see [2]) has conjectured that the Brauer-Manin obstruction is the only obstruction to the existence of global zero-cycles of degree one on $X$. In $\S 3$, we shall prove a very weak version of this conjecture.

TheOREM 1.3. Let $X$ be a projective, smooth geometrically integral variety over a number field $k$. Denote by $A$ the Albanese variety of $X$ and assume that

$$
\amalg^{1}(A)=\operatorname{ker}\left[\mathrm{H}^{1}(k, A) \rightarrow \bigoplus_{v} \mathrm{H}^{1}\left(k_{v}, A\right)\right]
$$

is finite. If $Z_{0}^{\mathrm{a}}(X)^{\mathrm{E}(X)} \neq \emptyset$ then $\widetilde{P}_{X}=1$.

Thus the Brauer-Manin obstruction is the only obstruction to the generic period being 1 . The proof makes use of semi-Albanese torsors, and thus depends in an essential way on a result due to Harari and Szamuely [7, Theorem 1.1].

Remark 1.4. We note that $P_{U}$ can indeed be larger than $P_{X}$ for $U$ open in $X$. For example, if $X$ is a proper curve of genus 0 , then via the anti-canonical embedding it can be written as a conic in $\mathbb{P}^{2}$ :

$$
X: a X^{2}+b Y^{2}=c Z^{2} .
$$


Hence the index is either 1 or 2 , and it is 1 exactly when we have a rational point. Now, removing two points at infinity, we obtain

$$
U: a x^{2}+b y^{2}=c,
$$

which is a torsor under a torus. Because $P_{U}$ divides $I$, it is either 1 or 2 , and because the torsor is trivial exactly when $P_{U}$ is 1 , we see that $P_{U}=I$. Hence in this case we have $\widetilde{P}=I$. However, since the Albanese of $X$ is trivial, $P_{X}$ is certainly 1 . In general one can show that for smooth curves over any field, the two invariants $\widetilde{P}$ and $I$ are the same.

To the authors' knowledge, not very much is known about the quotient $I / \widetilde{P}$, but in general it is not always 1 . For arbitrary varieties, even over a number field, the invariants are not equal. Indeed, one can construct varieties with rational points exactly when they have a zero-cycle of degree one, without 5-obstruction and without rational points. O. Wittenberg informs the first author that the del Pezzo surface of degree 4

$$
v w=x^{2}-5 y^{2}, \quad(v+w)(v+2 w)=x^{2}-5 z^{2},
$$

examined by Birch and Swinnerton-Dyer in [1] provides such an example. In this example, by Theorem $1.3, I=2$ and $\widetilde{P}=1$.

Furthermore, in [20], O. Wittenberg relates the generic period to more classical invariants. He shows among other things that for a smooth proper geometrically integral variety $X$ over a number field $k$ such that $X\left(\mathbb{A}_{k}\right) \neq \emptyset$ and the Tate-Shafarevich group of the Picard variety of $X$ over $k$ is finite, there is an equivalence between the following statements (see ibid., Theorem 3.3.1):

(a) $X\left(\mathbb{A}_{k}\right)^{\mathrm{E}} \neq \emptyset$;

(b) the elementary obstruction (see [18, Definition 2.3.5]) of $X$ vanishes;

(c) the generic period $\widetilde{P}$ is 1 .

REMARK 1.5. In [11] it is claimed but not proved that over a global field $k$ and a principally polarized abelian variety $A$ over $k$, there is the Cassels-Tate pairing (defined in [11])

$$
\amalg^{1}(A) \times \amalg^{1}(A) \rightarrow \mathbb{Q} / \mathbb{Z},
$$

which is perfect modulo maximal divisible subgroups. Having such a result in the function field case is exactly what is lacking to prove that the BrauerManin obstruction associated to $\mathrm{B}$ is the only one for abelian varieties over function fields. If we would have this one could extend Theorem 1.1 to any global field. In a personal communication B. Poonen informs the first author that to his knowledge there is no written proof of this fact in the function field case. 
2. A short proof of S. Saito's theorem. In this section we give our proof of Theorem 1.1. We let $C$ be a geometrically integral, smooth projective curve over a number field $k$, without any $\mathrm{5}(C)$-obstruction to zero-cycles of degree one. Also suppose that $\amalg^{1}(A)$ is finite, where $A$ is the Jacobian variety $\mathrm{Pic}_{C / k}^{0}$ of $C$. Recall that there is a uniquely defined morphism $p: C \rightarrow \operatorname{Pic}_{C / k}^{1}$, where $\operatorname{Pic}_{C / k}^{1}$ is a torsor under $A$, and that the morphism is universal with respect to morphisms into torsors under abelian varieties. See Theorem 3.2 for the statement of the existence over a perfect field (well known for curves) and a reference for the proof, or the discussion in [16, Chapter V, Section 23] for the case of a general field. By functoriality of the pairings, if $C$ has no $\mathrm{B}(C)$-obstruction to the existence of zero-cycles of degree one, then $\mathrm{Pic}_{C / k}^{1}$ does not have any obstruction associated to $\mathrm{S}\left(\mathrm{Pic}_{C / k}^{1}\right)$ for zero-cycles of degree one. By the following lemma this implies that $\operatorname{Pic}_{C / k}^{1}\left(\mathbb{A}_{k}\right)^{\mathrm{D}} \neq 0$.

Lemma 2.1. Let $k$ be a number field and $X$ a smooth geometrically integral variety over $k$ such that $X$ has a point in every completion $k_{v}$ of $k$. Then $X\left(\mathbb{A}_{k}\right)^{\mathrm{E}} \neq \emptyset$ if and only if $Z_{0}^{\mathrm{a}}(X)^{\mathrm{b}} \neq \emptyset$.

Proof. One direction is trivial and we prove the other one. It is well known that if $X$ as in the statement of the lemma has a point locally everywhere, there is an adelic point $Q=\left(Q_{v}\right)_{v} \in X\left(\mathbb{A}_{k}\right)$. A restrictioncorestriction argument shows that $i_{v}\left(\alpha\left(z_{v}\right)\right)=\operatorname{deg}\left(z_{v}\right) i_{v}\left(\alpha\left(Q_{v}\right)\right)$ for any zero-cycle $z_{v}$ on $X_{v}$, for $\alpha$ locally constant (i.e. in $\mathrm{Б}(X)$ ). Hence we can replace all zero-cycles of degree one with the adelic point $Q$, which will be orthogonal to $\mathrm{B}(X)$.

Going back to the situation preceding the lemma, in view of a result of Manin, by finitude of $\amalg^{1}(A), \operatorname{Pic}_{C / k}^{1}$ has a $k$-rational point (see Theorem 6.2.3 of [18] for a proof, or the original article of Manin [8]).

Denote by $\mathrm{CH}_{0}(X)$ the usual 0th Chow group of a variety $X$, that is, the full group of zero-cycles modulo rational equivalence, and recall that $\bar{K}$ denotes a separable closure of $K$. We now record the following general (well known) fact:

Lemma 2.2 ([10, Prop. 2.5]). Let $X$ be a smooth, proper and geometrically integral variety over a global field $K$ and assume that $X$ has a zero-cycle of degree one locally everywhere. Then $\operatorname{Pic}(X) \simeq \operatorname{Pic}(\bar{X})^{\Gamma_{K}}$. If $X$ is a curve, then in particular, $\mathrm{CH}_{0}(X)=\operatorname{Pic}(X)$ surjects onto the $K$-points of the Picard scheme $\operatorname{Pic}_{X / K}(K)=\operatorname{Pic}(\bar{X})^{\Gamma_{K}}$.

Proof. We include a proof for completeness. The Hochschild-Serre spectral sequence provides us with the exact sequence

$$
0 \rightarrow \operatorname{Pic}(X) \rightarrow \operatorname{Pic}(\bar{X})^{\Gamma_{K}} \rightarrow \operatorname{Br} K \stackrel{j}{\rightarrow} \operatorname{Br} X .
$$


If $X$ has a $K$-point, this point splits the map $j$ and so $j$ is injective. By a restriction-corestriction argument the same stays true if $X$ has a zero-cycle of degree one. Global class field theory tells us that the map $\operatorname{Br} K \rightarrow \bigoplus_{v} \operatorname{Br} K_{v}$ is injective. The condition that we have a zero-cycle locally everywhere implies that $\bigoplus_{v} \operatorname{Br} K_{v} \rightarrow \bigoplus_{v} \operatorname{Br} X_{v}$ is injective, and one concludes that $\operatorname{Pic}(X) \rightarrow \operatorname{Pic}(\bar{X})^{\Gamma_{K}}$ must be surjective, and hence bijective.

We have a natural identification $\mathrm{Pic}_{C / k}=\bigsqcup_{n \in \mathbb{Z}} \mathrm{Pic}_{C / k}^{n}$, where $\mathrm{Pic}_{C / k}^{n}$ denotes (cf. [12, Section 1.2]) for $n>0$ the $n$th Baer sum of torsors, for $n=0$ the trivial torsor and for negative $n$ it is defined by twisting the sum $-n$ times by the automorphism given by multiplication by -1 . This comes with a natural map $\overline{\operatorname{deg}}: \operatorname{Pic}_{C / k}(k) \rightarrow \mathbb{Z}$, sending an element in $\operatorname{Pic}_{C / k}^{n}(k)$ to $n \in \mathbb{Z}$, and the following diagram is commutative:

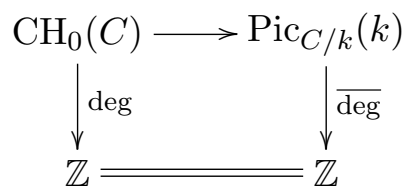

Since the upper horizontal map is a bijection under the hypothesis that there is a zero-cycle of degree one in every completion $k_{v}$, we see that $\overline{\operatorname{deg}}$ is surjective whenever deg is, and thus with the assumption that we have an adelic zero-cycle of degree one orthogonal to $\mathrm{S}(C)$, we see that the index is 1 and Theorem 1.1 is proved.

3. Brauer-Manin obstruction and generic periods. In this section we give the proof of the main theorem, Theorem 1.3, roughly as follows. After recalling the definition of the semi-Albanese torsor we first show that under the right conditions the period is equal to 1 (Lemma 3.4). We then recall that the group of locally constant elements is invariant under restriction to Zariski open sets (Lemma 3.5), and finally put this together to prove Theorem 1.3. The main technical tool is a recent result on the Hasse principle for semiabelian varieties by Harari and Szamuely [7].

First, let $U$ be a quasi-projective, smooth, geometrically integral variety over a field $k$. Recall that a semiabelian variety is a commutative group variety which is an extension of an abelian variety by a torus. Suppose we are given a $k$-morphism $p: U \rightarrow S^{1}$ where $S^{1}$ is a torsor under a semiabelian variety $S^{0}$ over $k$, with the following universal property: Given any $k$-morphism $m: U \rightarrow T^{1}$, where $T^{1}$ is a torsor under a semiabelian variety $T^{0}$ over $k$, there is a unique $k$-morphism $f^{1}: S^{1} \rightarrow T^{1}$ such that $h \circ p=f^{1}$, and a unique $k$-morphism of algebraic groups $f^{0}: S^{0} \rightarrow T^{0}$ such that $f^{1}$ is $f^{0}$-equivariant. This clearly determines the quadruple $\left(U, S^{0}, S^{1}, p\right)$, if it exists, up to unique isomorphism. 
Definition 3.1. A quadruple $\left(U, S^{0}, S^{1}, p\right)$ as above is a semi-Albanese torsor of $U$, and $S^{0}$ is called the semi-Albanese variety of $U$.

Suppose that $k$ is perfect. The following is a formal consequence of the existence of a semi-Albanese torsor over an algebraically closed field [17, Theorem 7] and the descent theory of [16, p. 112, 4.22], which was already remarked in [12]:

THEOREM 3.2. Let $U$ be a quasi-projective smooth variety over a perfect field $k$. Then a semi-Albanese torsor exists.

REMARK 3.3. If $X$ is also proper the semi-Albanese variety is the Albanese variety of $X$, and the semi-Albanese torsor is an "Albanese torsor", i.e. it is universal with respect to morphisms into torsors under abelian varieties. In this case it is desirable to write it as $\mathrm{Alb}_{X}^{1}$ instead. In general, if $X_{c}$ denotes a smooth compactification of $X$, then if $\mathrm{H}^{1}\left(X_{c}, \mathcal{O}_{X_{c}}\right)=0$, the abelian variety part of the semi-Albanese variety of $X$ is trivial and the semi-Albanese torsor is a torsor under a torus and is universal with respect to morphisms to torsors under tori.

Lemma 3.4. Let $V$ be a torsor under a semiabelian variety $S$ which is an extension of an abelian variety $A$ by a torus $T$, defined over a number field $k$, and suppose that $\mathrm{W}^{1}(A)$ is finite. Then the obstruction associated to $\mathrm{5}(V)$ for zero-cycles of degree one is the only one for rational points on $V$. That is, if $Z_{0}^{\mathrm{a}}(V)^{\mathrm{E}(V)} \neq \emptyset$ then $V(k) \neq \emptyset$.

Proof. Since $V$ is a torsor under an abelian group and has a zero-cycle of degree one locally everywhere, it actually has a $k_{v}$-rational point for every place $v$ and we can apply Lemma 2.1. The statement we now want to prove is well known for rational points whenever $S$ is an abelian variety (see [18, Theorem 6.2.3] for a proof, or the original article of Manin [8]) or a torus (see [18, Theorem 6.2.1]). The result for $\mathrm{Б}(V)$ in the case of arbitrary $S$ is due to Harari and Szamuely, [7].

We record the following lemma of invariance of $\mathrm{B}$ under restriction to open subsets.

Lemma 3.5 ([14, Lemma 6.1] or [6, Theorem 2.1.1]). Let $k$ be a number field and let $X$ be a smooth proper geometrically integral variety over $k$. Suppose $U$ is a non-empty Zariski open set in $X$. Then

$$
\mathrm{Б}(U)=\mathrm{Б}(X) .
$$

We now turn to:

Proof of Theorem 1.3. Let $X$ be as in the theorem. Let $U$ be an open set of $X$, and let $p: U \rightarrow \mathrm{SAlb}_{U}^{1}$ be its semi-Albanese torsor. Since by the above lemma, $\mathrm{\textrm {C }}(U)=\mathrm{\textrm {C }}(X)$, and elements of these groups are locally constant, 
$U$ has no $\mathrm{Б}(U)$-obstruction for zero-cycles of degree one. By functoriality of the Brauer-Manin pairing, the same holds true for $\mathrm{SAlb}_{U}^{1}$. Because of the finiteness assumption on $\amalg^{1}(A)$, Lemma 3.4 implies that the torsor is trivial, and so $P_{U}$, the period for $U$, is 1 . This is true for any open $U$ in $X$, and hence the generic period is 1 .

REMARK 3.6. Suppose now that $U$ is a torsor under a torus, and $X$ a smooth compactification thereof. If $X$ has no $\mathrm{B}(X)$-obstruction associated to zero-cycles of degree one, by Theorem 1.3 the period of $U$ is 1 (this rests only on [18, Theorem 6.2.1] and does not utilize the full result of [7]). But then it has a point and we recover a result by Colliot-Thélène and Sansuc saying that the Brauer-Manin obstruction is the only one for smooth compactifications of $k$-torsors under tori (see [18, Theorem 6.3.1], and the remark afterwards saying that we only need to consider locally constant elements). In any case, the generic period contains more information than the period associated to the mere $X$. An interesting question (suggested by Colliot-Thélène) would be to calculate the generic period of (a smooth compactification of) a non-abelian algebraic group and compare it to its index.

4. Alternative description of the period. In this section we give an additional description of the period as the cokernel of a map deg : $\mathrm{h}_{0}(\bar{X})^{\Gamma_{k}} \rightarrow \mathbb{Z}$. Suppose $k$ is perfect. The semi-Albanese scheme of $X \rightarrow$ Spec $k$ is the $k$-group scheme

$$
\underline{\mathrm{SAlb}}_{X / k}=\coprod_{n \in \mathbb{Z}} \operatorname{SAlb}_{X}^{n}
$$

where $\operatorname{SAlb}_{X}^{n}$ is defined as at the end of Section 2. In $[12,1.2]$, Ramachandran shows that this is a group scheme with various functorial and universal properties. We mimic his approach: We have an obvious $\Gamma_{k}$-equivariant map $X(\bar{k}) \rightarrow \operatorname{SAlb}_{X}^{1}(\bar{k})$, and we define a map from the group $Z_{0}(\bar{X})$ of zero-cycles to the $\bar{k}$-points of the semi-Albanese scheme to be the unique group homomorphism whose restriction to $X(\bar{k})$ is the above map. Taking Galois invariants gives a homomorphism of groups $Z_{0}(X) \rightarrow \underline{\mathrm{SAlb}}_{X / k}(k)$. By naturality and the fact that $S\left(\mathbb{A}_{k}^{1}\right)=S(k)$ for semiabelian varieties $S$ we see that this map factors over the Suslin homology group $\mathrm{h}_{0}(X)$ (see Appendix for definition of Suslin homology and argue as in [19, Lemma 3.1]).

Hence, there is a canonical homomorphism $\mathrm{h}_{0}(X) \rightarrow \underline{\mathrm{SAlb}}_{X / k}(k)$ such that the restriction to degree 0 is the generalized Albanese map (which we will refer to simply as the "Albanese map") of [19], and the structural morphism $X \rightarrow$ Spec $k$ induces the following commutative diagram (where 
the exactness on the left of the first line is our definition of $\left.\mathrm{h}_{0}(X)^{0}\right)$ :

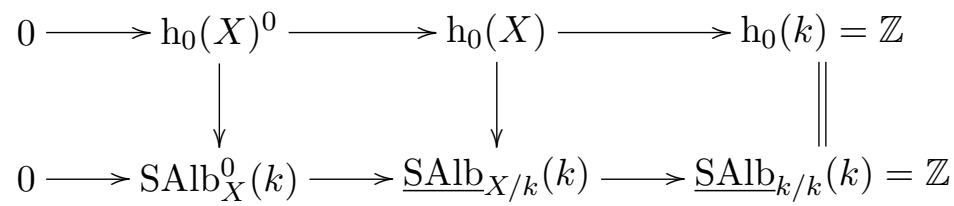

Definition 4.1. Let $X$ be a quasi-projective smooth variety over a perfect field $k$. We define

$$
S_{X}=\#\left\{\text { coker deg }: \mathrm{h}_{0}(\bar{X})^{\Gamma_{k}} \rightarrow \mathbb{Z}\right\} .
$$

TheOREM 4.2. Let $X$ be a quasi-projective, smooth geometrically integral variety over a field of characteristic 0 . Then $S_{X}=P_{X}$.

Proof. By the preceding remarks we have the following commutative diagram of Galois modules with exact rows:

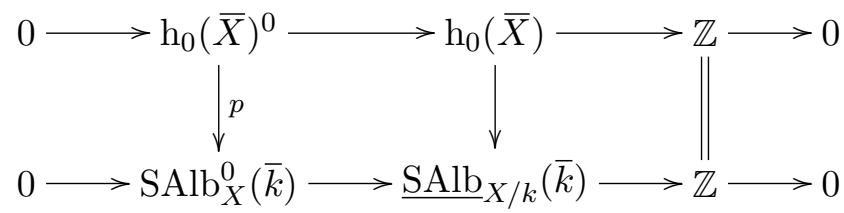

Here $p$ is the Albanese map (loc. cit.). Taking Galois cohomology gives the diagram

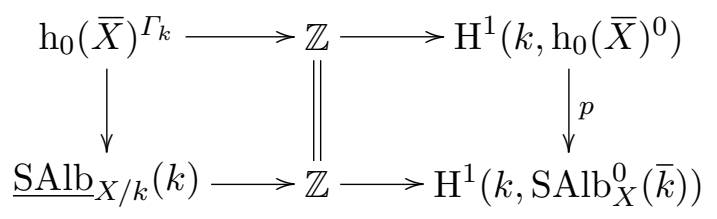

The image of 1 in $\mathrm{H}^{1}\left(k, \mathrm{SAlb}_{X}^{0}\right)$ is represented by the cocycle $\sigma \mapsto x_{0}^{\sigma}-x_{0}$ for $x_{0} \in \operatorname{SAlb}_{X}^{1}(\bar{k})$, i.e. it is the class of the semi-Albanese torsor. Now, the generalized theorem of Roitman can be formulated as saying that under the conditions of the theorem, the Albanese map $\mathrm{h}_{0}(\bar{X})^{0} \stackrel{p}{\longrightarrow} \underline{\mathrm{SAlb}}_{X / k}^{0}(\bar{k})$ is surjective with uniquely divisible kernel. This is the main theorem of Spiess and Szamuely [19]. It is established for a smooth connected variety $X$ admitting a smooth projective connected compactification over an arbitrary algebraically closed field of characteristic $p$ (for $p \geq 0$ ) and states that the Albanese map is an isomorphism on the prime-to- $p$ torsion subgroups. Since we are in characteristic 0 , the smooth projective compactification is provided by Hironaka. Finally, because for a uniquely divisible Galois module $M, \mathrm{H}^{i}(k, M)=0$ for $i>0$, the rightmost vertical homomorphism is an isomorphism, which finishes the proof. 
A. Appendix - Suslin homology, $\mathrm{h}_{0}$. In this section we recall some basic properties of the Suslin homology group $\mathrm{h}_{0}$. Let $X$ and $Y$ be any separated schemes of finite type over a field $k$. If $Y$ is connected, an elementary finite correspondence from $Y$ to $X$ over $k$ is an integral closed subscheme $Z$ of $X \times_{k} Y$, finite and surjective over $Y$. A finite correspondence between $X$ and $Y$ is a formal $\mathbb{Z}$-linear sum of elementary finite correspondences, and we denote the group of such as $\operatorname{Cor}(Y, X)$. Any closed subscheme $Z$ of $X \times_{k} Y$ finite and surjective over $Y$ defines a finite correspondence by associating to it the correspondence $\sum n_{i} Z_{i, \text { red }}$ where the sum is over irreducible components $Z_{i}$ of $Z$ such that $Z_{i \text {,red }}$ is surjective over $Y$, and $n_{i}$ is the geometric multiplicity of $Z_{i \text {,red }}$ in $Z$ (compare [9, Construction 1.3]). If $Y=\bigsqcup_{\alpha} Y_{\alpha}$ is the decomposition of $Y$ into its connected components, one defines $\operatorname{Cor}(Y, X)=\bigoplus_{\alpha} \operatorname{Cor}\left(Y_{\alpha}, X\right)$. Note that the set of finite correspondences from $\operatorname{Spec} k$ to $X$, $\operatorname{Cor}(\operatorname{Spec} k, X)$, is just the group of zero-cycles on $X$.

If $P$ is a $k$-point of $Y$ and $Z$ is a closed subscheme of $X \times_{k} Y$, denote by $Z(P)$ the scheme-theoretic fiber of $Z$ over $X=X \times_{k} P$. Consider the points 0 and 1 of $\mathbb{A}_{k}^{1}$ and define a map $\operatorname{Cor}\left(\mathbb{A}_{k}^{1}, X\right) \rightarrow \operatorname{Cor}(\operatorname{Spec} k, X)$ by

$$
Z \mapsto Z(0)-Z(1)
$$

We define $\mathrm{h}_{0}(X)$, the 0th Suslin homology of $X$, to be the group of zerocycles on $X$ modulo the group generated by the finite correspondences coming from $\mathbb{A}_{k}^{1}$ to $X$ in the above sense. We note the following properties, which are not difficult to show.

Proposition A.1. Let $X, Y$ be two separated schemes of finite type over a field $k$. Then the following holds:

(a) Let $\mathrm{CH}_{0}(X)$ be the 0th Chow group. If the structural morphism $X \rightarrow$ Spec $k$ is proper, then there is a map $\mathrm{h}_{0}(X) \rightarrow \mathrm{CH}_{0}(X)$ which is moreover an isomorphism.

(b) $\mathrm{h}_{0}$ is covariantly functorial with respect to morphisms $f: X \rightarrow Y$.

(c) The degree map mapping a zero-cycle $\sum n_{i} P_{i}$ to $\sum n_{i}\left[k\left(P_{i}\right): k\right]$ factors over $\mathrm{h}_{0}(X) \rightarrow \mathrm{h}_{0}(k)=\mathbb{Z}$ where the map $\mathrm{h}_{0}(X) \rightarrow \mathrm{h}_{0}(k)$ is given by the structural morphism $X \rightarrow$ Spec $k$.

For lack of a specially tailored reference, we include the following proofs:

Proof. Let $V$ be a dimension 1 integral closed subscheme of $X \times_{k} \mathbb{P}_{k}^{1}$ which is dominant on the second factor. Then $[V(0)]-[V(1)]$ is a zero-cycle on $X$ and it follows as in [4, Proposition 1.6] that rational equivalence on zero-cycles is generated by the relation determined by all such $V$. Now, if $Z \subseteq X \times_{k} \mathbb{A}^{1}$ is an elementary finite correspondence, its closure $\widetilde{Z}$ in $X \times_{k} \mathbb{P}_{k}^{1}$ defines such an object. This shows there is always a map $\mathrm{h}_{0}(X) \rightarrow \mathrm{CH}_{0}(X)$. Suppose $X \rightarrow \operatorname{Spec} k$ is moreover proper. A closed integral subscheme $\widetilde{Z} \subseteq$ 
$X \times_{k} \mathbb{P}_{k}^{1}$ which is dominant over $\mathbb{P}_{k}^{1}$ is proper over Spec $k$ by virtue of $X$ being proper, and if $\widetilde{Z}$ is moreover of dimension one it is finite over $\mathbb{P}_{k}^{1}$. As such it determines a closed integral subscheme $Z \subseteq X \times_{k} \mathbb{A}_{k}^{1}$ which is a finite elementary correspondence from $\mathbb{A}_{k}^{1}$ to $X$. This is inverse to the above operation and proves (a).

Now, let $f: X \rightarrow Y$ be a morphism of separated schemes of finite type over a Noetherian base $S$. If $Z \subseteq X$ is a closed integral subscheme, finite and surjective over $S$, then the schematic image $f(Z)$ is a closed integral subscheme of $Y$, finite and surjective over $S$ (see [9, Lemma 1.4]). If $Y$ is connected, the pushforward is defined as $f_{*}(Z)=d f(Z)$ where $d=$ $[k(Z): k(f(Z))]$, which is finite, and the definition for general $Y$ is similar. The above rule thus assigns a homomorphism $f_{*}: \operatorname{Cor}(V, X) \rightarrow \operatorname{Cor}(V, Y)$ to a separated scheme $V$ of finite type over $S$. Putting $S=\mathbb{A}_{k}^{1}$ or Spec $k$ we obtain pushforwards on $\operatorname{Cor}\left(\mathbb{A}_{k}^{1},-\right)$ respectively $\operatorname{Cor}(\operatorname{Spec} k,-)$, and they clearly respect the obvious compatibility conditions for restricting to the points 0 and 1 , so we obtain a homomorphism

$$
f_{*}: \mathrm{h}_{0}(X) \rightarrow \mathrm{h}_{0}(Y) .
$$

The last point is now a consequence of the definition of the degree map.

Acknowledgements. The first author would like to thank D. Harari, who was his advisor during the writing of this article, for interesting remarks, discussions, careful reading of the manuscript and continued encouragement. He would also like to thank J.-L. Colliot-Thélène and O. Wittenberg for stimulating conversations, and B. Poonen for answering a question on his paper with M. Stoll [11]. The second author would like to thank J. Milne for helpful conversations. We would also like to thank the referee for a detailed report suggesting several now implemented improvements of the text.

V. Scharaschkin was partially supported by NSF Grant 0070850 and UQ Early Career Researcher 2003001483.

\section{References}

[1] B. J. Birch and H. P. F. Swinnerton-Dyer, The Hasse problem for rational surfaces, J. Reine Angew. Math. 274/275 (1975), 164-174.

[2] J.-L. Colliot-Thélène, Conjectures de type local-global sur l'image des groupes de Chow dans la cohomologie étale, in: Algebraic K-theory (Seattle, WA, 1997), Proc. Sympos. Pure Math. 67, Amer. Math. Soc., 1999, 1-12.

[3] - Un théorème de finitude pour le groupe de Chow des zéro-cycles d'un groupe algébrique linéaire sur un corps p-adique, Invent. Math. 159 (2005), 589-606.

[4] W. Fulton, Intersection Theory, Springer, 1998.

[5] A. Grothendieck, Le groupe de Brauer III, in: Dix exposés sur la cohomologie des schémas, North-Holland, Amsterdam, and Masson, Paris, 1968. 
[6] D. Harari, Méthode des fibrations et obstruction de Manin, Duke Math. J. 75 (1994), 221-260.

[7] D. Harari and T. Szamuely, Local-global principles for 1-motives, ibid. 143 (2008), 531-557.

[8] Yu. I. Manin, Le groupe de Brauer-Grothendieck en géométrie diophantienne, in: Actes du Congrès International des Mathématiciens (Nice, 1970), Vol. 1, 1970, 401-411.

[9] C. Mazza, V. Voevodsky and C. Weibel, Lectures on Motivic Cohomology, Clay Math. Monogr. 2, Amer. Math. Soc., 2006.

[10] J. S. Milne, Comparison of the Brauer group with the Tate-Šafarevič group, J. Fac. Sci. Univ. Tokyo Sect. IA 28 (1982), 735-743.

[11] B. Poonen and M. Stoll, The Cassels-Tate pairing on polarized abelian varieties, Ann. of Math. 150 (1999), 1109-1149.

[12] N. Ramachandran, Duality of Albanese and Picard 1-motives, K-Theory 22 (2001), 271-301.

[13] S. Saito, Some observations on motivic cohomology of arithmetic schemes, Invent. Math. 98 (1989), 371-404.

[14] J.-J. Sansuc, Groupe de Brauer et arithmétique des groupes algébriques linéaires sur un corps de nombres, J. Reine Angew. Math. 327 (1981), 12-80.

[15] J.-P. Serre, Corps locaux, Hermann, Paris, 1962.

[16] —, Groupes algébriques et corps de classes, Hermann, Paris, 1959.

[17] —, Morphismes universels et variétés d'Albanese, in: Exposés de séminaires 19501989, Soc. Math. France, 2001, 141-160.

[18] A. N. Skorobogatov, Torsors and Rational Points, Cambridge Univ. Press, 2001.

[19] M. Spiess and T. Szamuely, On the Albanese map for smooth quasi-projective varieties, Math. Ann. 325 (2003), 1-17.

[20] O. Wittenberg, On Albanese torsors and the elementary obstruction, ibid. 340 (2008), 805-838.

Department of Mathematical Sciences

University of Tokyo

Tokyo, Japan

E-mail: eriksson@ms.u-tokyo.ac.jp
Department of Mathematics University of Queensland St. Lucia 4072, Qld, Australia E-mail: victors@maths.uq.edu.au

Received on 1.7.2006

and in revised form on 21.8.2008 